\title{
Cardiomyocyte differentiation of perinatally-derived mesenchymal stem cells
}

\author{
KUNEERAT NARTPRAYUT ${ }^{1}$, YAOWALAK U-PRATYA ${ }^{2}$, PAKPOOM KHEOLAMAI $^{3}$, SIRIKUL MANOCHANTR $^{3}$, \\ METHICHIT CHAYOSUMRIT ${ }^{4}$, SURAPOL ISSARAGRISIL $^{2,4}$ and AUNGKURA SUPOKAWEJ ${ }^{1}$ \\ ${ }^{1}$ Department of Clinical Microscopy, Faculty of Medical Technology; ${ }^{2}$ Division of Hematology, \\ Department of Medicine, Faculty of Medicine Siriraj Hospital, Mahidol University, Bangkok; \\ ${ }^{3}$ Division of Cell Biology, Department of Pre-Clinical Sciences, Faculty of Medicine, Thammasat University, \\ Pathumthani; ${ }^{4}$ Siriraj Center of Excellence for Stem Cell Research, Faculty of Medicine, \\ Siriraj Hospital, Mahidol University, Bangkok, Thailand
}

Received October 29, 2012; Accepted February 20, 2013

DOI: $10.3892 / \mathrm{mmr} .2013 .1356$

\begin{abstract}
Coronary heart disease is major cause of mortality worldwide and several risk factors have been shown to play a role in its pathogenesis, including smoking, obesity, hypertension and hypercholesterolemia. A number of therapeutic methods have been developed to improve the quality of patients' lives, including stem cell therapy using mesenchymal stem cells (MSCs). Perinatal sources, including the placenta (PL) and umbilical cord (UC), are rich sources of MSCs and have been identified as a potential source of cells for therapeutic use. Their role in cardiogenic differentiation is also of contemporary medical interest. The present study demonstrated the induced differentiation of MSCs obtained from the UC, PL and Wharton's jelly (WJ) into cardiomyocytes, using $10 \mu \mathrm{M}$ 5-azacytidine. The characteristics of the MSCs from each source were studied and their morphology was compared. An immunofluorescence analysis for the cardiac-specific markers, GATA4 and Troponin T (TnT), was performed and tested positive in all sources. The expression of the cardiac-specific genes, Nkx2.5, $\alpha$-cardiac actin and TnT, was analyzed by real-time RT-PCR and presented as fold change increases. The expression of each of the markers was observed to be higher in the 5-azacytidine-treated MSCs. The differences in expression among the sources of treated MSCs was as follows: TnT had the highest level of expression in the bone marrow (BM) MSCs; $\alpha$-cardiac actin had the highest level of expression in
\end{abstract}

Correspondence to: Dr Aungkura Supokawej, Department of Clinical Microscopy, Faculty of Medical Technology, 2 Prannok Road, Siriraj, Bangkok-Noi, Bangkok 10700, Thailand

E-mail: mtajr@mahidol.ac.th

Abbreviations: BM, bone marrow; BSA, bovine serum albumin; DMEM, Dulbecco's modified Eagle's medium; GAPDH, glyceraldehyde 3-phosphate dehydrogenase; MSC, mesenchymal stem cell; PL, placenta; TnT, troponin T; UC, umbilical cord; WJ, Wharton's jelly.

Key words: 5-azacytidine, cardiomyocyte, differentiation, mesenchymal stem cell, placenta, umbilical cord the PLMSCs; and all the genes were expressed at significantly high levels in the WJMSCs compared with the control group. The present study showed the ability of alternative perinatally-derived MSCs to differentiate into cardiomyocyte-like cells and how this affects the therapeutic use of these cells.

\section{Introduction}

Mesenchymal stem cells (MSCs) are a type of adult stem cell that have attracted significant attention due to their unique properties. The first source of human MSCs was identified and isolated from bone marrow (BM) (1). The BMMSCs showed capacity for self-renewal and multipotent differentiation. The potential uses of MSCs have been studied extensively in vitro and in animal models (2-4). MSCs are hypothesized to have a high potential for use in tissue engineering and regenerative medicine $(5,6)$. However, the availability of BMMSCs is limited due to the location of the cells. BM aspiration, or biopsy, is an invasive surgery, and the the differentiation capacity varies relative to the age of the donor (7). Optional MSC sources have been identified, including adipose tissue (8), umbilical cord blood (9) and peripheral blood (10) and Wharton's jelly (WJ) (11). The most noteworthy source of MSCs is the perinatally-derived stem cells i.e., stem cells from the placenta (PL), amniotic fluid and umbilical cord (UC). These stem cells have a number of advantages, including their ability to be easily obtained, a low immunogenicity and lack of ethical concerns (12). MSCs may be isolated and cultured from the UC, PL and WJ (13).

Coronary heart disease is the third leading cause of mortality worldwide. A number of risk factors have been revealed to play a role in the pathogenesis of this disease, including smoking, obesity, hypertension and hypercholesterolemia. The main mechanism is the blockage of blood flow through the heart leading to myocardial infarction (the death of cardiac muscle cells). Cell therapy is one of a number of therapeutic methods developed to improve the quality of life of patients suffering from heart problems. Various cell types have been studied extensively to determine the most effective outcome. Adult stem cells are one of the candidates for cell therapy. MSCs are the best option due to three important 
properties; multilineage differentiation, possible autologous sources and their immunoregulatory role. The transdifferentiation of MSCs has been studied extensively and it is widely accepted that MSCs are multipotent. The role of MSC differentiation into cardiomyocyte-like cells has been demonstrated in animal models and in vitro studies (14-16). The present study analyzed the cardiomyocyte differentiation ability of MSCs from perinatal sources in comparison with BMMSCs.

\section{Materials and methods}

Perinatal cell culture. The present study was approved by the Siriraj Institution Review Board (SIRB). Human UCs and PLs were collected from full-term deliveries and immersed in sterile phosphate-buffered saline (PBS) supplemented with $100 \mathrm{U} / \mathrm{ml}$ penicillin and $100 \mu \mathrm{g} / \mathrm{ml}$ streptomycin under sterile conditions. The WJ, PLs and UCs were stored and processed separately. The samples were washed twice using PBS prior to their preparation into $2-3-\mathrm{cm}^{2}$ sections. Perinatal tissues were collected in $15 \mathrm{ml}$ centrifugal tubes containing PBS and then washed twice under centrifugation at $984 \mathrm{x} \mathrm{g}$ for $5 \mathrm{~min}$. The pellets from the washing procedure were digested using $0.5 \%$ trypsin-EDTA at $37^{\circ} \mathrm{C}$ for $30 \mathrm{~min}$ and placed in $25-\mathrm{cm}^{2}$ tissue culture flasks in Dulbecco's modified Eagle's medium (DMEM; Gibco-BRL, Carlsbad, CA, USA) supplemented with $10 \%$ fetal bovine serum (FBS), $100 \mathrm{U} / \mathrm{ml}$ penicillin and $100 \mu \mathrm{g} / \mathrm{ml}$ streptomycin. The tissues were incubated under $5 \% \mathrm{CO}_{2}$ until MSC outgrowth occurred from the explant. The complete medium was changed twice a week. Subpassaging was performed using $0.5 \%$ trypsin-EDTA to expand the quantity of cells used in the experiment.

BMMSCs. BM aspirations were obtained from healthy donors following written informed consent. The BM aspiration was collected in 5,000 IU sodium heparin and diluted by PBS at a 1:1 ratio prior to the isolation of mononuclear cells. Following the method of Boyum (17), the cell suspension was layered on Ficoll-Hypaque at a 1:1 ratio followed by centrifugation at $984 \mathrm{x}$ g for $30 \mathrm{~min}$. Mononuclear cells were harvested from the interphase layer into new centrifugal tubes for 2 cycles of washing. The cells were seeded in T25 flasks using DMEM supplemented with $10 \% \mathrm{FBS}, 100 \mathrm{U} / \mathrm{ml}$ penicillin and $100 \mu \mathrm{g} /$ $\mathrm{ml}$ streptomycin. The culture supernatant was removed 5-7 days later and the adherent cells were maintained at $37^{\circ} \mathrm{C}$ with $5 \%$ $\mathrm{CO}_{2}$. The BMMSCs from passages 2-5 were used in this study.

\section{MSC characterization}

Criteria. The cells from the BM and perinatal sources were characterized as MSCs based on the following criteria: plastic adherence, positive and negative specific cell surface markers and multi-potential differentiation (13).

MSC surface markers. The cell surface markers for the MSCs were examined using a flow cytometer (FACSCalibur ${ }^{\mathrm{TM}}$, Becton-Dickinson, Franklin Lakes, NJ, USA). The immunophenotype of the MSCs was characterized using the following mouse monoclonal antibodies: cluster of differentiation CD34-PE, CD45-FITC, CD73-PE (all purchased from BD Pharmingen, San Diago, CA, USA), CD90-FITC, CD105-FITC and CD106-PE (all purchased from AbD SeroTec, Raleigh, NC, USA). The MSCs between passages 2 and 5 were harvested using $0.5 \%$ trypsin-EDTA. A $5 \times 10^{5}$ cell suspension (50 $\left.\mu \mathrm{l}\right)$ was incubated at $4^{\circ} \mathrm{C}$ for $30 \mathrm{~min}$ in the dark with mouse anti-human antibodies. Following incubation, the cells were washed twice with cold PBS and centrifuged at $984 \mathrm{x}$ g for $5 \mathrm{~min}$ at $4^{\circ} \mathrm{C}$. The cells were then fixed with $300 \mu 11 \%$ paraformaldehyde prior to analysis by a flow cytometer (Becton-Dickinson).

Osteogenic and adipogenic differentiation of MSCs. The MSCs from passages 2-5 were cultured in Advance STEM Osteogenic Differentiation medium (OsteoDiff) for osteoblast differentiation (Hyclone Laboratories, Inc., Logan, UT, USA) and Advance STEM Adipogenic Differentiation medium (AdipoDiff; Hyclone Laboratories) for adipocyte differentiation. The MSC cells were seeded at a density of $1 \times 10^{5}$ cells $/ \mathrm{ml}$ and maintained in each differentiation medium. The media were changed twice a week. The differentiated conditioned MSCs were maintained for 3 weeks, at which time their osteogenic and adipogenic properties were evaluated using immunocytochemical staining with alkaline phosphatase and Oil Red O (Sigma-Aldrich, St. Louis, MO, USA).

In vitro treatment with 5-azacytidine. The MSCs obtained from the BM, UC, PL and WJ during passages 2-5 were used in this experiment. The MSCs were seeded at densities of $1 \times 10^{5}$ cells in DMEM supplemented with $10 \% \mathrm{FBS}, 100 \mathrm{U} / \mathrm{ml}$ penicillin and $100 \mu \mathrm{g} / \mathrm{ml}$ streptomycin for $24 \mathrm{~h}$. The treatment group was treated with $10 \mu \mathrm{M}$ 5-azacytidine (Sigma-Aldrich) in complete medium for $24 \mathrm{~h}$ subsequent to which it was changed into complete medium for 3 weeks. The morphology, growth rate and viability were then observed.

RNA preparation and real-time RT-PCR. RNA was collected from the control and 5-azacytidine-treated groups at weeks 1 and 3. The total RNA was isolated from the cells using TRIzol reagent (Invitrogen Life Technologies, Carlsbad, CA, USA) and quantified by spectrophotometry at $260 \mathrm{~nm}$. The total RNA $(2 \mu \mathrm{g})$ was used for reverse transcription with the first-strand cDNA synthesis kit (Invitrogen Life Technologies). Real-time PCR was performed for 40 cycles using the ABI 7500 real-time PCR system (Applied Biosystems, Bedford, MA, USA) and analyses for cardiac- and myogenic-specific genes were performed using the following primers: $\alpha$-cardiac actin (260 bp), sense 5'-TCTATGAGGGCTACGCTTTG-3' and antisense 5'-GCCAATAGTGATGACTTGGC-3'; Troponin T (TnT; 225 bp), sense 5'- AGAGCGGAAAAGTGGGAAGA-3' and antisense 5'-CTGGTTATCGTTGATCCTGT-3'; Nkx2.5 (136 bp), sense 5'-CTGCCGCCGCCAACAAC -3' and antisense 5'-CGCGGGTCCCTTCCCTACCA-3'; and glyceraldehyde 3-phosphate dehydrogenase (GAPDH; 139 bp) sense 5'-GTCAACGGATTTGGTCGTATTG-3' and antisense 5'-CATGGGTGGAATCATATTGGAA-3'. Each cycle consisted of denaturation at $95^{\circ} \mathrm{C}$ for $10 \mathrm{sec}$, annealing at $60^{\circ} \mathrm{C}$ for $10 \mathrm{sec}$ and extension at $72^{\circ} \mathrm{C}$ for $40 \mathrm{sec}$. The products were quantified by the comparative $\mathrm{C}_{t}$ method. Samples were compared with the control group, normalized against GAPDH and presented as fold change increases.

Immunofluorescence staining. The MSCs from passages 2-5 were cultured on chamber slides (Lab-Tek, Thermo Fisher Scientific, Rochester, NY, USA) at $1 \times 10^{5}$ cells/well with DMEM supplemented with $10 \%$ FBS, $100 \mathrm{U} / \mathrm{ml}$ penicillin and 


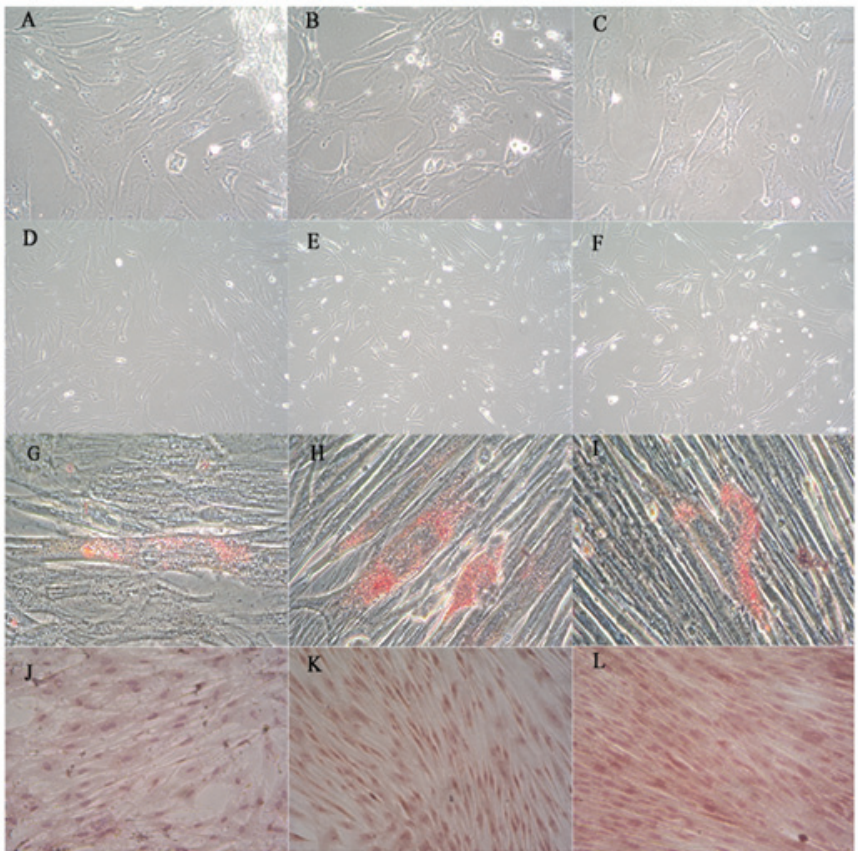

Figure 1. Morphology and characterization of perinatal MSCs. Perinatal MSCs from the tissues were observed to be spindle-shaped; (A) UCMSCs, (B) WJMSCs and (C) PLMSCs. Following subpassaging of all MSC sources, the morphology was fibroblast-like; (D) UCMSCs, (E) WJMSCs and (F) PLMSCs. Following culturing of perinatal MSCs in adipogenic medium for 3 weeks, the cells were stained with Oil Red $\mathrm{O}$ and small fat droplets were revealed in the cytoplasm; (G) UCMSCs, (H) WJMSCs and (I) PLMSCs. Alkaline phosphatase staining was used to characterize osteogenic differentiation and all MSC sources showed positive staining; (J) UCMSCs, (K) WJMSCs and (L) PLMSCs (magnification, x100). MSCs, mesenchymal stem cells; UC, umbilical cord; PL, placenta; WJ, Wharton's jelly
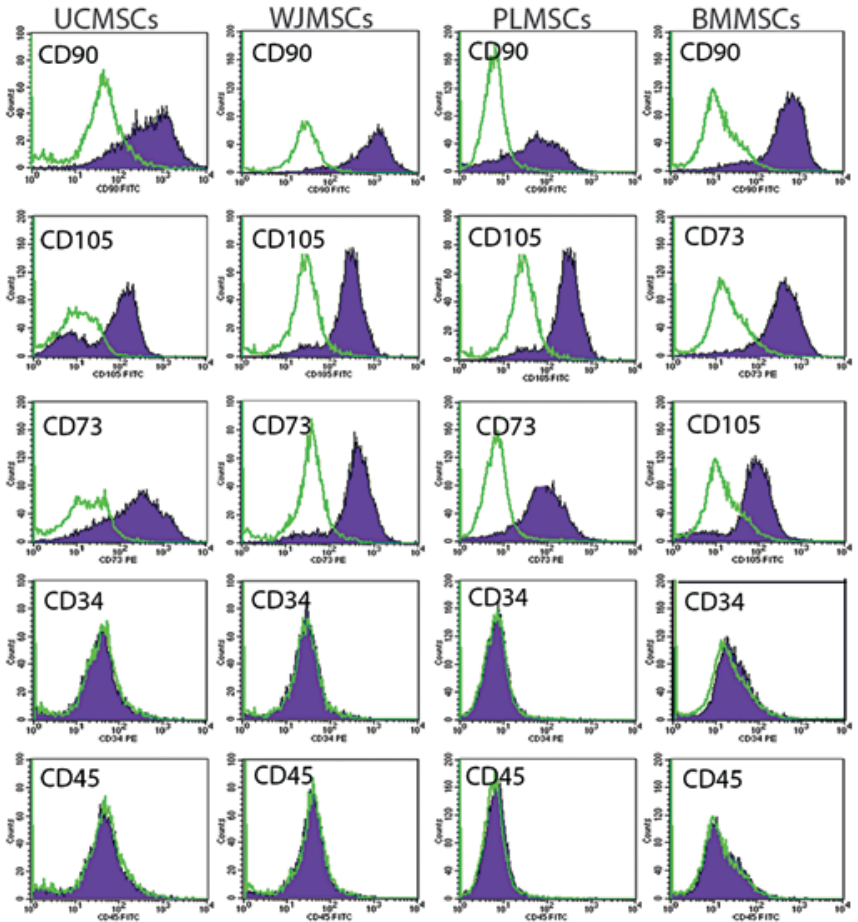

Figure 2. Cell surface markers of MSCs. UCMSCs, WJMSCs, PLMSCs and BMMSCs were found to be positive for the MSC markers CD105, CD90 and CD73 and negative for the hematopoietic markers CD34 and CD45. MSCs, mesenchymal stem cells; UC, umbilical cord; PL, placenta; WJ, Wharton's jelly; BM, bone marrow; CD, cluster of differentiation.
$100 \mu \mathrm{g} / \mathrm{ml}$ streptomycin. The $10 \mu \mathrm{M} 5$-azacytidine treatment and control groups from days 3 and 7 were used for immunofluorescence analysis. The cells were washed twice with PBS prior to fixation using $4 \%$ paraformaldehyde in $\mathrm{PBS}$ at $4^{\circ} \mathrm{C}$ for $30 \mathrm{~min}$. Non-specific binding was blocked using $4 \%$ bovine serum albumin (BSA), followed by incubation with purified goat polyclonal TnT and mouse monoclonal GATA4 primary antibodies (both Santa Cruz Biotechnology, Inc., Santa Cruz, CA, USA). The samples were then washed twice with PBS and overlaid with rabbit anti-mouse FITC- (Millipore, Billerica, MA, USA) and donkey anti-goat PE-conjugated secondary antibodies (Santa Cruz Biotechnology, Inc.) for $30 \mathrm{~min}$ at $4^{\circ} \mathrm{C}$ in the dark. The samples were washed twice, followed by DAPI counterstaining and mounting using ProLong Gold Antifade (Invitrogen Life Technologies).

Statistical analysis. The data were analyzed using SPSS version 11.5 (SPSS, Inc., Chicago, IL). Data are expressed as the mean \pm SEM. The significance was analyzed using the Mann-Whitney $\mathrm{U}$ test. $\mathrm{P}<0.05$ was considered to indicate a statistically significant difference.

\section{Results}

Morphology and characterization of perinatal MSCs. The UCMSCs, PLMSCs and WJMSCs were observed to exhibit different morphologies following tissue culture for 1 week. There were limited morphological differences between the tissue sources (Fig. 1A-C). The cells continued to grow and reached confluence within week 2 of the primary culture. Subpassages of MSCs grown in complete medium revealed a spindle-shaped cell morphology (Fig. 1D-F). The MSCs from passages 3 and 4 were characterized for osteogenic-adipogenic differentiation capacity and cell surface markers. Following culture of the MSCs in adipogenic and osteogenic media for 3 weeks, cell-specific cytochemical staining was performed. The UCMSCs, PLMSCs and WJMSCs were positive for Oil Red O (Fig. 1G-I) and alkaline phosphatase staining (Fig. 1J-L). The MSCs were shown to be positive for MSC surface markers CD73, CD90 and CD105 and negative for hematopoietic cell surface markers CD34 and CD45 (Fig. 2).

Effect of 5-azacytidine on the MSCs. The UCMSCs, WJMSCs and PLMSCs from passage 3 were used at this stage of the study. The MSCs were treated with $10 \mu \mathrm{M} 5$-azacytidine for $24 \mathrm{~h}$ and maintained in complete medium for 3 weeks. The morphology, cell number and percent viability from week 1 were observed and compared against the control group. The viability of all the MSC sources following treatment with 5-azacytidine was $94-100 \%$ in the UCMSCs; $84-100 \%$ in the WJMSCs and 93-100\% in the PLMSCs. The morphology of the treated cells revealed similarities with the control group in all MSC sources subsequent to being cultured for 6 days (data not shown). The cell numbers in the 5-azacytidine-treated group were lower than those of the control group during the initial days in all the MSC sources.

Immunofluorescence study of cardiomyocyte markers during MSC differentiation. The UCMSCs, WJMSCs, PLMSCs and BMMSCs from passages 3-5 were used at this stage of the 


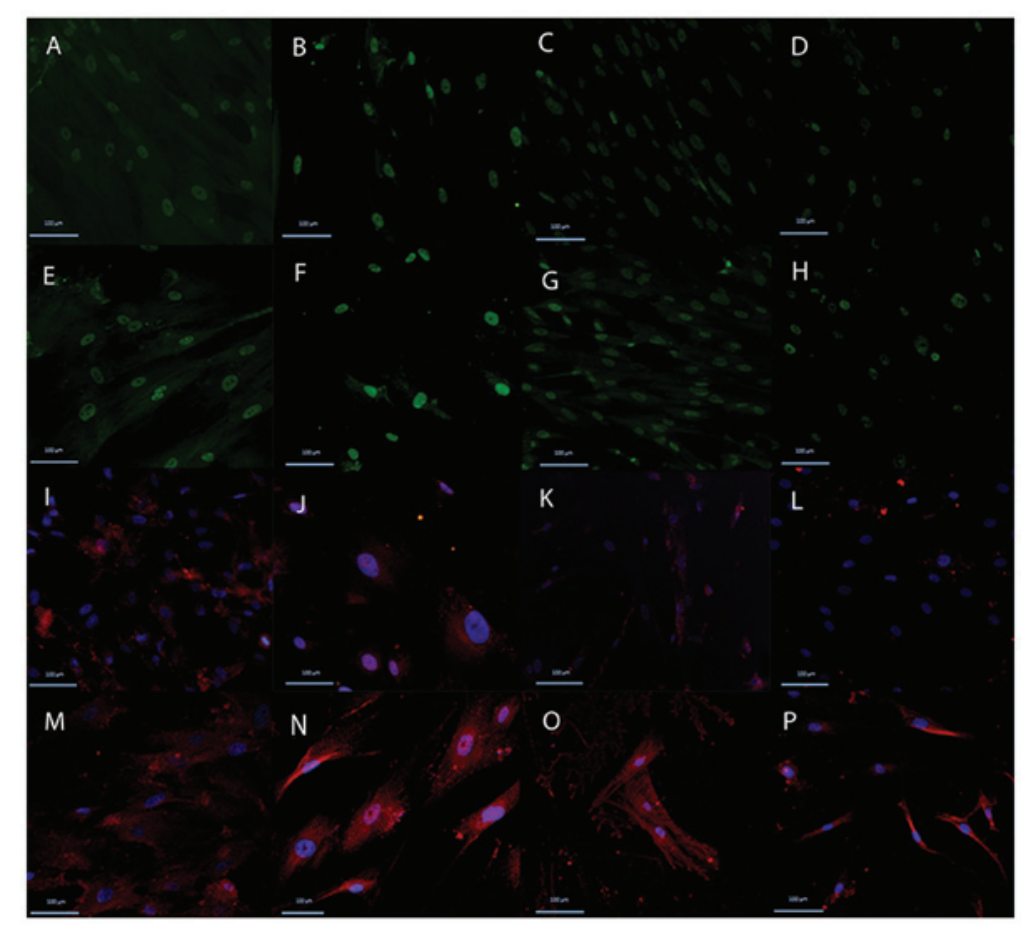

Figure 3. Immunofluorescence images of MSCs labeled with the cardiac-specific antibodies (A-H) GATA4 (green) and (I-P) cardiac TnT (red). The 5-azacytidine treated (E and M) UCMSCs, (F and N) WJMSCs, (G and O) PLMSCs and (H and P) BMMSCs revealed a marked expression when compared with (A-D and I-L) the controls following 1 week of culturing. The nuclei were counterstained with DAPI (magnification, x100). TnT, Troponin T; MSCs, mesenchymal stem cells; UC, umbilical cord; PL, placenta; WJ, Wharton's jelly; BM, bone marrow.

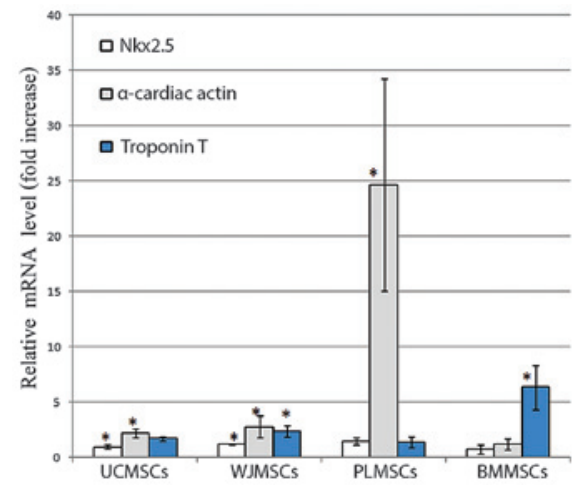

Figure 4. Expression of the cardiac-specific genes $\mathrm{Nkx} 2.5, \alpha$-cardiac actin and cardiac TnT was determined by real-time RT-PCR. Expression of each cardiac-specific genes from all MSC sources was compared with the contro as fold changes. Data were obtained from three independent experiments and presented as the mean \pm SEM. ${ }^{*} \mathrm{P}<0.05,5$-azacytidine-treated vs. control cells. TnT, Troponin T; MSC, mesenchymal stem cell.

study. The MSCs were treated with $10 \mu \mathrm{M} 5$-azacytidine for $24 \mathrm{~h}$ and then changed into complete medium for 7 days. The cardiac-specific markers, GATA4 and cardiac TnT, were examined by immunofluorescence staining. The treated and control MSCs revealed GATA4 expression in the nucleus, green in Fig. 3E-H. The cardiac TnT in the cytoplasm is presented in red in Fig. 3M-P. The expression of the two markers was higher in the treated cells compared with the control.

Expression of cardiomyocyte-specific genes following treatment with 5-azacytidine. The mRNA expression of the cardiomyocyte specific markers $\mathrm{Nkx} 2.5$, $\alpha$-cardiac actin and cardiac TnT, was examined in the BMMSCs,
UCMSCs, PLMSCs and WJMSCs following treatment with $10 \mu \mathrm{M} 5$-azacytidine for $24 \mathrm{~h}$ and maintenance in culture for 1-3 weeks. The quantitative analysis of the expression of the cardiac-specific markers was performed by real-time RT-PCR and reported as a fold increase of the control. The cardiac-specific markers showed a higher expression level than that of the controls in the samples treated with 5-azacytidine for $24 \mathrm{~h}$ and cultured for 1 week (Fig. 4). The expression decreased in samples maintained for 2 and 3 weeks (data not shown).

\section{Discussion}

BMMSCs have been studied extensively and a number of promising medicinal applications have been reported. However, at present, the clinical utilization of BMMSCs is limited due to the invasiveness of the harvesting procedure (18). A new source of MSCs has been identified that demonstrates a similar potential to BMMSCs $(8,9,11)$. MSCs from perinatal tissue (amniotic membrane, UC and PL) were identified as potential sources of MSCs due to their immunogenic property, non-invasive sourcing and absence of ethical issues (11). Moreover, the unlimited differentiation potential of perinatal MSCs has been identified and extensively studied $(19,20)$.

The present study aimed to develop cardiomyocyte-like cells from perinatal MSCs and compare them with BMMSCs. The UC, WJ and PL were isolated and explants of each were cultured for their residing MSCs. The MSCs were treated with the hypomethylating agent, 5-azacytidine, and the cardiomyocyte characteristics were evaluated. Expression of the cardiomyocyte markers was analyzed by real-time RT-PCR and an increased expression of $\mathrm{Nkx} 2.5$, TnT and $\alpha$-cardiac actin was observed in the 5-azacytidine-treated MSCs 
compared with the controls. However, the cardiomyocyte gene expression patterns varied depending on the source of the MSCs. These observations may be due to variations in the capacity and fate of each MSC source (21), which is determined by the epigenetic state of the cells $(22,23)$.

The effect of 5-azacytidine in the present study was consistent with previous studies $(18,24,25)$. However, the mechanism of this hypomethylating agent yielded a non-specific pattern and remains undefined. In addition, a reduction in cardiac-specific marker expression was identified following 5-azacytidine treatment for 2-3 weeks. The transient expression of these markers may be a limitation of using 5-azacytidine as a differentiation initiation factor. The combination of 5-azacytidine with other factors may be more successful for improving the properties of cardiomyocyte-differentiated MSCs.

The role of MSCs in the regeneration of damaged tissue remains controversial. The mechanisms include the role of MSCs in neoangiogenesis (26,27), transdifferentiation (28), anti-inflammatory responses (29) and as a protective factor (30). The success of transplantation may be due to various aspects that play a concerted role in tissue regeneration. A clinical trial of MSCs in the treatment of cardiac ischemia revealed a reduced efficacy in the engraftment rate and functional capacity (31). The majority of studies concerning BMMSCs have revealed that these cells are autologous and allogeneic. The allogeneic origin of MSCs from other sources may be optional and is particularly true of perinatal MSCs due to their potential role in growth and neonatal development and their availability. However, further studies are required to ensure the proper use of these MSCs for stem cell transplantation.

\section{Acknowledgements}

The present study was supported by grants from the Thailand Research Fund (no. RTA 488-0007) and the Commission on Higher Education (no. CHERES-RG-49).

\section{References}

1. Friedenstein AJ, Chailakhyan RK, Latsinik NV, Panasyuk AF and Keiliss-Borok IV: Stromal cells responsible for transferring the microenvironment of the hemopoietic tissues. Cloning in vitro and retransplantation in vivo. Transplantation 17: 331-340, 1974.

2. Pittenger MF, Mackay AM, Beck SC, et al: Multilineage potential of adult human mesenchymal stem cells. Science 284 : 143-147, 1999.

3. Wu Y, Chen L, Scott PG and Tredget EE: Mesenchymal stem cells enhance wound healing through differentiation and angiogenesis. Stem Cells 25: 2648-2659, 2007.

4. Morigi M, Introna M, Imberti B, et al: Human bone marrow mesenchymal stem cells accelerate recovery of acute renal injury and prolong survival in mice. Stem Cells 26: 2075-2082, 2008.

5. Fu X and Li H: Mesenchymal stem cells and skin wound repair and regeneration: possibilities and questions. Cell Tissue Res 335: 317-321, 2009.

6. Richardson SM, Hoyland JA, Mobasheri R, Csaki C, Shakibaei M and Mobasheri A: Mesenchymal stem cells in regenerative medicine: opportunities and challenges for articular cartilage and intervertebral disc tissue engineering. J Cell Physiol 222: 23-32, 2010.

7. Mueller SM and Glowacki J: Age-related decline in the osteogenic potential of human bone marrow cells cultured in three-dimensional collagen sponges. J Cell Biochem 82: 583-590, 2001.

8. Bunnell BA, Flaat M, Gagliardi C, Patel B and Ripoll C: Adipose-derived stem cells: isolation, expansion and differentiation. Methods 45: 115-120, 2008.
9. Lee OK, Kuo TK, Chen WM, Lee KD, Hsieh SL and Chen TH: Isolation of multipotent mesenchymal stem cells from umbilical cord blood. Blood 103: 1669-1675, 2004

10. Villaron EM, Almeida J, López-Holgado N, et al: Mesenchymal stem cells are present in peripheral blood and can engraft after allogeneic hematopoietic stem cell transplantation. Haematologica 89: 1421-1427, 2004

11. Wang HS, Hung SC, Peng ST, et al: Mesenchymal stem cells in the Wharton's jelly of the human umbilical cord. Stem Cells 22: 1330-1337, 2004.

12. Surbek D, Wagner A and Schoeberlein A: Perinatal stem cell therapy. In: Perinatal Stem Cells. Cetrulo CL, Cetrulo KJ and Cetrulo CL Jr (eds). John Wiley \& Sons, Hoboken, NJ, pp51-60, 2009.

13. Dominici M, Le Blanc K, Mueller I, et al: Minimal criteria for defining multipotent mesenchymal stromal cells. The International Society for Cellular Therapy position statement. Cytotherapy 8: 315-317, 2006.

14. Shim WS, Jiang S, Wong $\mathrm{P}$, et al: Ex vivo differentiation of human adult bone marrow stem cells into cardiomyocyte-like cells. Biochem Biophys Res Commun 324: 481-488, 2004.

15. Hattan N, Kawaguchi $\mathrm{H}$ ando K, et al: Purified cardiomyocytes from bone marrow mesenchymal stem cells produce stable intracardiac grafts in mice. Cardiovasc Res 65: 334-344, 2005.

16. Kruglyakov PV, Sokolova IB, Zin'kova NN, et al: In vitro and in vivo differentiation of mesenchymal stem cells in the cardiomyocyte direction. Bull Exp Biol Med 142: 503-506, 2006.

17. Boyum A: Isolation of leucocytes from human blood. A two-phase system for removal of red cells with methylcellulose as erythrocyte-aggregating agent. Scand J Clin Lab Invest Suppl 97: 9-29, 1968

18. Xu W, Zhang X, Qian H, et al: Mesenchymal stem cells from adult human bone marrow differentiate into a cardiomyocyte phenotype in vitro. Exp Biol Med (Maywood) 229: 623-631, 2004.

19. Tsai MS, Hwang SM, Tsai YL, Cheng FC, Lee JL and Chang YJ: Clonal amniotic fluid-derived stem cells express characteristics of both mesenchymal and neural stem cells. Biol Reprod 74: 545-551, 2006.

20. Wu KH, Zhou B, Lu SH, et al: In vitro and in vivo differentiation of human umbilical cord derived stem cells into endothelial cells. J Cell Biochem 100: 608-616, 2007.

21. Kadivar M, Khatami S, Mortazavi Y, Shokrgozar MA, Taghikhani $\mathbf{M}$ and Soleimani $\mathbf{M}$ : In vitro cardiomyogenic potential of human umbilical vein-derived mesenchymal stem cells. Biochem Biophys Res Commun 340: 639-647, 2006.

22. Benayahu D, Shefer G and Shur I: Insights into the transcriptional and chromatin regulation of mesenchymal stem cells in musculo-skeletal tissues. Ann Anat 191: 2-12, 2009.

23. Collas P: Epigenetic states in stem cells. Biochim Biophys Acta 1790: 900-905, 2009.

24. Martin-Rendon E, Sweeney D, Lu F, Girdlestone J, Navarrete C and Watt SM: 5-Azacytidine-treated human mesenchymal stem/ progenitor cells derived from umbilical cord, cord blood and bone marrow do not generate cardiomyocytes in vitro at high frequencies. Vox Sang 95: 137-148, 2008.

25. Zhang Y, Chu Y, Shen W and Dou Z: Effect of 5-azacytidine induction duration on differentiation of human first-trimester fetal mesenchymal stem cells towards cardiomyocyte-like cells. Interact Cardiovasc Thorac Surg 9: 943-946, 2009.

26. Nagaya N, Kangawa K, Itoh T, et al: Transplantation of mesenchymal stem cells improves cardiac function in a rat model of dilated cardiomyopathy. Circulation 112: 1128-1135, 2005.

27. Silva GV, Litovsky S, Assad JA, et al: Mesenchymal stem cells differentiate into an endothelial phenotype, enhance vascular density, and improve heart function in a canine chronic ischemia model. Circulation 111: 150-156, 2005.

28. Shake JG, Gruber PJ, Baumgartner WA, et al: Mesenchymal stem cell implantation in a swine myocardial infarct model: engraftment and functional effects. Ann Thorac Surg 73: 1919-1926, 2002.

29. Guo J, Lin GS, Bao CY, Hu ZM and Hu MY: Anti-inflammation role for mesenchymal stem cells transplantation in myocardial infarction. Inflammation 30: 97-104, 2007.

30. Markel TA, Wang Y, Herrmann JL, et al: VEGF is critical for stem cell-mediated cardioprotection and a crucial paracrine factor for defining the age threshold in adult and neonatal stem cell function. Am J Physiol Heart Circ Physiol 295: H2308-H2314, 2008.

31. Copland IB and Galipeau J: Death and inflammation following somatic cell transplantation. Semin Immunopathol 33: 535-550, 2011. 